\title{
Neurotoxicity due to prophylactic cranial irradiation for small-cell lung cancer: A retrospective analysis
}

\author{
YOSHIRO NAKAHARA ${ }^{1,2}$, YUSUKE TAKAGI ${ }^{1}$, YUSUKE OKUMA ${ }^{1}$, YUKIO HOSOMI $^{1}$, \\ TATSURU OKAMURA ${ }^{1}$, MASAHIKO SHIBUYA ${ }^{1}$ and NORIYUKI MASUDA ${ }^{2}$ \\ ${ }^{1}$ Department of Thoracic Oncology and Respiratory Medicine, Tokyo Metropolitan Cancer and Infectious Diseases Center, \\ Komagome Hospital, Tokyo 113-8677; ${ }^{2}$ Department of Respiratory Medicine, Kitasato University School \\ of Medicine, Sagamihara, Kanagawa 252-0239, Japan
}

Received December 17, 2014; Accepted April 24, 2015

DOI: $10.3892 / \mathrm{mco} .2015 .581$

\begin{abstract}
Prophylactic cranial irradiation (PCI) is an established part of standard therapy for small-cell lung cancer (SCLC). However, the concerns regarding severe late neurotoxicity following PCI have not yet been systematically investigated. Therefore, the aim of this study was to investigate the neurocognitive functioning of SCLC patients treated with PCI. Limited-disease SCLC (LD-SCLC) patients $(\mathrm{n}=40)$ treated at Tokyo Metropolitan Cancer and Infectious Diseases Center Komagome Hospital (Tokyo, Japan) between January, 2004 and December, 2011 were retrospectively reviewed. A total of $18 \mathrm{LD}$-SCLC patients were treated with PCI (median age, 65.5 years; range, 52-75 years), whereas 22 LD-SCLC patients did not receive PCI (median age, 65.5 years; range, 57-84 years). The median follow-up for PCI and non-PCI patients was 22 months (range, 4-85 months) and 14.5 months (range, 2-49 months), respectively. Brain metastases occurred in 6 (33\%) PCI patients and $11(50 \%)$ non-PCI patients. In the PCI group, dementia occurred in 5 of the $12 \mathrm{PCI}$ patients without brain metastases $(42 \%, 3-40$ months after $\mathrm{PCI}$ ) and in 1 of the 11 non-PCI patients without brain metastases (9\%, 4 months after initial treatment). The frequency of dementia in the PCI group was significantly higher compared with that in the non-PCI group $(\mathrm{P}=0.0357)$. In the PCI group, all the patients who developed dementia were aged $>65$ years (range, 66-75 years). Gait disturbance appeared in 25\% of the PCI patients without brain metastases (9-27 months after PCI); these patients were also aged $>65$ years. Patients aged $>65$ years were significantly more likely to develop dementia $(\mathrm{P}=0.0028)$
\end{abstract}

Correspondence to: Dr Yoshiro Nakahara, Department of Thoracic Oncology and Respiratory Medicine, Tokyo Metropolitan Cancer and Infectious Diseases Center, Komagome Hospital, 3-18-22 Honkomagome, Bunkyo-ku, Tokyo 113-8677, Japan E-mail: md100062@cick.jp

Key words: neurotoxicity, prophylactic cranial irradiation, small-cell lung cancer, dementia, gait disturbance and gait disturbance $(\mathrm{P}=0.0291)$. Therefore, neurotoxicity due to PCI tends to appear more frequently in older patients.

\section{Introduction}

Small-cell lung cancer (SCLC) exhibits several characteristics that distinguish it from other types of lung cancer, namely the risk of early hematogenous dissemination, its marked sensitivity to chemotherapy and radiation therapy and its particular propensity to metastasize to the brain. The frequency of brain metastases in SCLC at diagnosis is $\sim 10 \%$; however, brain metastases may occur in $>50 \%$ of 2 -year survivors (1). As cytotoxic drugs have difficulty crossing the blood-brain barrier, the effectiveness of chemotherapy for brain metastases is unsatisfactory.

To evaluate the effectiveness of prophylactic cranial irradiation (PCI) to control micrometastases to the brain, a number of clinical trials have been performed (2-4). A meta-analysis demonstrated that, in patients with limited disease (LD) who achieved a complete response (CR) with initial chemoradiotherapy (CRT), PCI decreased the risk of brain metastases (from 58.8 to $33.3 \%$ at 3 years) and increased the 3-year survival rate (from 15.3\% in the control group to 20.7\%) (5). Therefore, PCI has been established as a part of standard therapy for LD-SCLC. Furthermore, the European Organization for Research and Treatment of Cancer (EORTC) trial reported that PCI decreased the risk of symptomatic brain metastases and prolonged overall survival in extensive disease (ED) that responded to chemotherapy (6). A pooled analysis reported that PCI was associated with a significant survival benefit in LD-SCLC as well as ED-SCLC patients (7). Based on the results of those studies, PCI is currently considered a part of standard therapy for ED-SCLC. However, hair loss, skin erythema, headache, nausea and vomiting, appetite loss, fatigue, dizziness and drowsiness have been reported as early adverse effects due to PCI, while muscle weakness, dementia, gait disturbance and leukoencephalopathy have been reported as late PCI-associated adverse toxicities. The number of studies that have attempted to evaluate the effect of PCI on neurocognitive functioning (NCF) and other quality-of-life (QOL) markers is currently limited. Therefore, the aim of this study was to investigate the effect of PCI on NCF in patients with SCLC. 


\section{Patients and methods}

Patients. This retrospective study enrolled LD-SCLC patients treated at the Tokyo Metropolitan Cancer and Infectious Diseases Center, Komagome Hospital (Tokyo, Japan) between January, 2004 and December, 2011.

This study was approved by the Institutional Review Board of Tokyo Metropolitan Cancer and Infectious Diseases Center Komagome Hospital. As all the data were collected anonymously, the requirement for individual patient consent was waived.

PCI. PCI was delivered with the use of two opposed lateral fields using 6-MV X-rays. The following schedules for cranial irradiation were used: 25 Gy in 10 fractions; 26 Gy in 13 fractions; 27 Gy in 15 fractions; 30 Gy in 15 fractions; or 36 Gy in 18 fractions. In LD cases, PCI was initiated 4-5 weeks after the first day of the last cycle of concurrent CRT, or 4-8 weeks after the last radiotherapy in sequential CRT.

Assessment. NCF was assessed using the Hasegawa Dementia Scale-Revised (HDS-R), where possible. The HDS-R is a scale widely used for the evaluation of dementia and consists of nine items as follows: Patient's age (1 point); knowledge of date and place (6 points); recollection of 3 words ( 3 points); knowledge of arithmetic (2 points); recitation of figures in reverse (2 points); repetition of the last 3 words heard in sequence (6 points); memory of 5 objects (5 points); and knowledge of words for vegetables ( 5 points) $(8,9)$. The maximum total score is 30 and the total score is used for the analysis. The sensitivity and specificity of the HDS-R are 0.9 and 0.82 , respectively, when the cut-off point is set at $20 / 21$. If the score is $<21$, dementia is suspected (10). In the present study, dementia was defined as a score of $<21$ points on the HDS-R, or following diagnosis by the physicians in cases without HDS-R scores.

$\mathrm{NCF}$ and gait disturbance were also assessed according to the National Cancer Institute Common Terminology Criteria for Adverse Events (CTCAE, version 4.0; http://ctep.cancer.gov/ protocolDevelopment/electronic_applications/ctc.htm) from the patients' medical records. The characteristics of patients with and without dementia or gait disturbance were compared. Older patients were defined as those aged $>65$ years, as based on previous studies (11).

Statistical methods. Differences in characteristics were evaluated using the $\chi^{2}$ test. Survival was estimated using the Kaplan-Meier method. All the tests were two-sided, with a significance level of 0.05 . All the data were analyzed using JMP software, version 10 (SAS Institute, Cary, NC, USA).

\section{Results}

Patient characteristics. During the study period, a total of 40 LD-SCLC cases were identified, of whom 18 [14 men (78\%) and 4 women (22\%)] were treated with PCI, whereas 22 [12 men $(55 \%)$ and 10 women (45\%)] did not receive PCI. The median age of the PCI and non-PCI patients was 65.5 years (range, 52-75 years) and 71.5 years (range, 57-84 years), respectively, at the time of completion of the initial treatment. In the PCI group, 15 patients $(83 \%)$ were initially treated with concurrent
Table I. Patient characteristics.

\begin{tabular}{lcc}
\hline Characteristics & PCI $(\mathrm{n}=18)$ & Non-PCI $(\mathrm{n}=22)$ \\
\hline Median age, years (range) & $65.5(52-75)$ & $71.5(57-84)$ \\
Gender & & \\
$\quad$ Male & 14 & 12 \\
Female & 4 & 10 \\
Initial CRT & 15 & 6 \\
Concurrent & 3 & 16 \\
Sequential & & \\
Response to initial CRT & 11 & 4 \\
CR & 7 & 18 \\
PR & & \\
\hline
\end{tabular}

PCI, prophylactic cranial irradiation; CRT, chemoradiotherapy; CR, complete response; PR, partial response.

CRT and $3(17 \%)$ were treated with sequential CRT. In the non-PCI group, 6 patients $(27 \%)$ were initially treated with concurrent CRT and 16 (73\%) were treated with sequential CRT. In the PCI group, 11 patients $(61 \%)$ achieved a CR and $7(39 \%)$ achieved a partial response (PR) with initial treatment. In the non-PCI group, 4 patients (18\%) achieved a CR and $18(82 \%)$ a PR with initial treatment (Table I).

PCI was delivered as 25 Gy in 10 fractions in 6 patients, $26 \mathrm{~Gy}$ in 13 fractions in 2 patients, $27 \mathrm{~Gy}$ in 15 fractions in 1 patient, 30 Gy in 15 fractions in 8 patients and 36 Gy in 18 fractions in 1 patient (Table II).

Survival and brain metastases. The median follow-up for PCI and non-PCI patients was 22 months (range, 4-85 months) and 14.5 months (range, 2-49 months), respectively. In the PCI group, 6 patients (33\%) succumbed to lung cancer and 8 patients (44\%) remained alive without disease progression. Brain metastases occurred following PCI in 6 patients (33\%) (Table II). In the non-PCI group, 14 patients (64\%) succumbed to lung cancer and 3 patients (14\%) remained alive without disease progression. In this group, brain metastases occurred in 11 of the 22 patients $(50 \%)$ (Table III).

In the PCI group, the median overall survival and the median survival without brain metastasis were not reached. In the non-PCI group, the median overall survival and the median survival without brain metastasis were 20 and 9 months, respectively (Table III).

Neurocognitive function. Dementia occurred in 5 of the 12 patients without brain metastases $(42 \%)$ in the PCI group and in 1 of 22 patients $(5 \%)$ in the non-PCI group. Patients who received PCI were significantly more likely to develop dementia following treatment compared with patients who did not receive PCI $(\mathrm{P}=0.0357)$ (Table III). The median time between PCI and the appearance of dementia was 12 months (range, 3-40 months) (Table II). A total of 3 patients were diagnosed based on the deterioration of the HDS-R and 2 patients were diagnosed based on the attending physicians' assessment. The cognitive disturbances of these 5 patients were classified 
Table II. Treatment and outcomes in patients treated with PCI.

\begin{tabular}{|c|c|c|c|c|c|c|c|c|}
\hline Case & $\begin{array}{l}\text { Age (years)/ } \\
\text { gender }\end{array}$ & Response & $\begin{array}{l}\text { Radiation } \\
\text { dose } \\
(\mathrm{Gy} / \mathrm{fr})\end{array}$ & $\begin{array}{l}\text { Survival time } \\
\text { (months) }\end{array}$ & $\begin{array}{l}\text { Brain metastasis } \\
\text { (time after PCI, } \\
\text { months) }\end{array}$ & $\begin{array}{l}\text { HDS-R } \\
\text { (points) }\end{array}$ & $\begin{array}{c}\text { Dementia } \\
\text { (time after PCI, } \\
\text { months) }\end{array}$ & $\begin{array}{c}\text { Gait disturbance } \\
\text { (time after PCI, } \\
\text { months) }\end{array}$ \\
\hline 1 & $52 / \mathrm{M}$ & PR & $26 / 13$ & $>85$ & & 28 & & \\
\hline 2 & $55 / \mathrm{M}$ & $\mathrm{CR}$ & $30 / 15$ & $>41$ & & & & \\
\hline 3 & 55/M & PR & $30 / 15$ & $>44$ & & 28 & & \\
\hline 4 & $58 / \mathrm{M}$ & PR & $26 / 13$ & 19 & 5 & & & \\
\hline 5 & 59/M & PR & $30 / 15$ & $>39$ & & 30 & & \\
\hline 6 & 64/M & CR & $25 / 10$ & $>4$ & & 30 & & \\
\hline 7 & $64 / F$ & $\mathrm{CR}$ & $25 / 10$ & $>14$ & 8 & & & \\
\hline 8 & $65 / \mathrm{F}$ & $\mathrm{CR}$ & $25 / 10$ & $>14$ & & 30 & & \\
\hline 9 & 65/M & $\mathrm{CR}$ & $25 / 10$ & $>25$ & 8 & & & \\
\hline 10 & $66 / \mathrm{M}$ & PR & $30 / 15$ & 18 & 14 & & & \\
\hline 11 & $66 / \mathrm{M}$ & $\mathrm{CR}$ & $36 / 18$ & $>35$ & & 11 & 35 & 23 \\
\hline 12 & $68 / \mathrm{M}$ & PR & $30 / 15$ & $>40$ & & 19 & 40 & 27 \\
\hline 13 & $70 / \mathrm{M}$ & CR & $30 / 15$ & 30 & 10 & & & \\
\hline 14 & $72 / \mathrm{F}$ & $\mathrm{CR}$ & $30 / 15$ & $>42$ & & 16 & 12 & 9 \\
\hline 15 & $72 / \mathrm{M}$ & CR & $25 / 10$ & $>16$ & & 26 & & \\
\hline 16 & $75 / \mathrm{M}$ & PR & $25 / 10$ & $>5$ & & & 3 & \\
\hline 17 & $75 / F$ & $\mathrm{CR}$ & $27 / 15$ & 18 & 6 & & & \\
\hline 18 & $75 / \mathrm{M}$ & $\mathrm{CR}$ & $30 / 15$ & 8 & & & 5 & \\
\hline
\end{tabular}

PCI, prophylactic cranial irradiation; HDS-R, Hasegawa Dementia Scale-Revised; M, male; F, female; CR, complete response; PR, partial response.

Table III. Treatment outcomes in patients who received and those who did not receive PCI.

\begin{tabular}{lcrr}
\hline Outcomes & PCI $(\mathrm{n}=18)$ & non-PCI $(\mathrm{n}=22)$ & P-value \\
\hline Brain metastasis & 6 & 11 & 0.2866 \\
Dementia & 5 & 1 & 0.0357 \\
Gait disturbances & 3 & 0 & 0.0241 \\
Median survival time, months & $\mathrm{NR}$ & 20 & 0.0196 \\
\hline
\end{tabular}

PCI, prophylactic cranial irradiation; NR, not reached.

as grade 1 in 2 patients, grade 2 in 2 patients and grade 3 in 1 patient, according to the CTCAE.

In the PCI group, the median age of patients with dementia was 72 years (range, 66-75 years). Patients aged $>65$ years were significantly more likely to develop dementia $(\mathrm{P}=0.0028)$. The radiation doses administered to these 5 patients were $25 \mathrm{~Gy}$ in 10 fractions ( 1 patient), 30 Gy in 15 fractions ( 3 patients) and 36 Gy in 18 fractions (1 patient) (Table IV).

Gait disturbance appeared in 3 of the 12 PCI patients without brain metastases $(25 \%, 9-27$ months after PCI); no patients without brain metastases in the non-PCI group exhibited gait disturbances. Patients who received PCI were significantly more likely to develop gait disturbances $(\mathrm{P}=0.0241)$.

The gait disturbances in these 3 patients were classified as grade 2 in 1 patient and grade 3 in 2 patients, according to the CTCAE, version 4.0. The 3 patients were aged 66,68 , and
72 years. Patients treated with PCI who were aged $>65$ years were significantly more likely to develop gait disturbance $(\mathrm{P}=0.0291)$ (Table V).

\section{Discussion}

In this retrospective study, the PCI-related neurotoxicities following standard treatment for SCLC were reviewed, with a median follow-up time of 22 months. Following PCI, dementia occurred in $42 \%$ of all patients without brain metastases, whereas gait disturbances appeared in $25 \%$ of all patients. Patients who received PCI were significantly more likely to develop dementia and gait disturbances compared with patients who did not receive PCI. Among patients treated with PCI, those aged $>65$ years were significantly more likely to develop dementia and gait disturbances. 
Table IV. Patient characteristics according to the presence of dementia.

\begin{tabular}{|c|c|c|c|}
\hline Characteristics & Dementia $(n=5)$ & No dementia $(n=13)$ & $\mathrm{P}$-value \\
\hline Median age, years (range) & $72(66-75)$ & $64(52-75)$ & 0.0028 \\
\hline$(\leq 65)$ & 0 & 9 & \\
\hline$(>65)$ & 5 & 4 & \\
\hline Gender & & & 0.8873 \\
\hline Male & 4 & 10 & \\
\hline Female & 1 & 3 & \\
\hline Radiation dose, Gy/fr & & & 0.4435 \\
\hline $25 \mathrm{~Gy}$ & 1 & 5 & \\
\hline$>25 \mathrm{~Gy}$ & 4 & 8 & \\
\hline
\end{tabular}

Table V. Patient characteristics according to the presence of gait disturbance.

\begin{tabular}{|c|c|c|c|}
\hline Characteristics & Gait disturbance $(n=3)$ & No gait disturbance $(\mathrm{n}=15)$ & P-value \\
\hline Median age, years (range) & $68(66-72)$ & $64.5(52-75)$ & 0.0291 \\
\hline$(\leq 65)$ & 0 & 9 & \\
\hline$(>65)$ & 3 & 6 & \\
\hline Gender & & & 0.6255 \\
\hline Male & 2 & 12 & \\
\hline Female & 1 & 3 & \\
\hline Radiation dose, Gy/fr & & & 0.0988 \\
\hline 25 Gy & 0 & 6 & \\
\hline$>25 \mathrm{~Gy}$ & 3 & 9 & \\
\hline
\end{tabular}

The number of studies evaluating the effect of PCI on NCF and other QOL markers is currently limited. It remains controversial whether the neurotoxicities following PCI are clinically significant. Certain previous studies concluded that PCI caused no significant increase in the incidence of neurotoxicities. Grosshans et al (12) reported the results of neuropsychological tests prospectively conducted prior to and following PCI in 37 patients. In their study, there was no convincing evidence for long-term PCI-related neurotoxic effects (median follow-up, 23 months; range, 6-120 months). The median age of the patients enrolled in that study was 59 years. Arriagada et al conducted a prospective study of 300 SCLC patients randomly assigned to the PCI or non-PCI groups (2). A neuropsychological examination and a computed tomography scan of the brain were performed at the time of random assignment and repeatedly assessed after 6, 18, 30 and 48 months. There were no significant differences between the two groups in terms of neuropsychological function or abnormalities indicated by computed tomography brain scans. The mean age of the patients in the non-PCI and PCI groups was 56 and 57 years, respectively.

However, other studies reported that PCI possibly decreased $\mathrm{NCF}$ and that neurotoxicities were more common in older patients. Gondi et al reported that PCI was associated with a higher risk of decline in self-reported cognitive functioning and the Hopkins Verbal Learning Test-Recall and -Delayed Recall at 6 and 12 months in the pooled analysis of two Radiation Therapy Oncology Group trials (13). The median age of the study patients was 62 years. Moreover, a prospective study from Germany reported that patients aged $\geq 65$ years who received PCI exhibited a trend towards distinct changes in the white matter on brain magnetic resonance imaging (11). These analyses did not include East-Asian patients, although the life expectancy of the general population is quite long in Japan.

Our study demonstrated that PCI-related neurotoxicities were also more common among older patients in Japan. However, there were several limitations to our study. First, there were not enough baseline evaluations in terms of HDS-R, as this was a retrospective analysis. However, it was confirmed that all the patients had sufficient preserved NCF to understand the risks and the schedule of the treatment prior to receiving PCI. Thus, the dementia and gait disturbances observed in this study were not present prior to PCI. Second, this study included cases treated with various radiation doses for PCI. At present, the standard dose of PCI for LD-SCLC is considered to be $25 \mathrm{~Gy} / 10 \mathrm{fr}$, based on the results of a previous trial in which 25 Gy achieved significantly better survival compared with 36 Gy (14). Our study included old cases, prior to the establishment of the standard radiation dose in PCI. As a result, there were no significant differences in neurotoxicities among radiation doses in this analysis. In addition, this was a retrospective study with a small sample size. A prospective study with a large sample size is required to confirm our findings.

In conclusion, the adverse effects of PCI on NCF, particularly the risk of dementia and gait disturbances, tend to appear 
frequently in older patients. The treatment for SCLC in older patients should be designed considering the balance between benefits and risks, including late toxicities caused by PCI.

\section{References}

1. Glantz MJ, Choy H and Yee L: Prophylactic cranial irradiation in small cell lung cancer: rationale, results and recommendations. Semin Oncol 24: 477-483, 1997.

2. Arriagada R, Le Chevalier T, Borie F, et al: Prophylactic cranial irradiation for patients with small-cell lung cancer in complete remission. J Natl Cancer Inst 87: 183-190, 1995.

3. Gregor A, Cull A, Stephens RJ, et al: Prophylactic cranial irradiation is indicated following complete response to induction therapy in small cell lung cancer: result of a multicenter randomized trial. United Kingdom Coordinating Committee for Cancer Research (UKCCCR) and the European Organization for Research and Treatment of Cancer (EORTC). Eur J Cancer 33 1752-1758, 1997.

4. Laplanche A, Monnet I, Santos-Miranda JA, et al: Controlled clinical trial of prophylactic cranial irradiation for patients with small-cell lung cancer in complete remission. Lung Cancer 21: 193-201, 1998

5. Auperin A, Arriagada R, Pignon JP, et al: Prophylactic cranial irradiation for patients with small-cell lung cancer in complete remission. N Engl J Med 341: 476-484, 1999.

6. Slotman B, Faivre-Finn C, Kramer G, et al: Prophylactic cranial irradiation in extensive small-cell lung cancer. N Engl J Med 357: 664-672, 2007.

7. Schild SE, Foster NR, Meyers JP, et al; North Central Cancer Treatment Group: Prophylactic cranial irradiation in small-cell lung cancer: findings from a North Central Cancer Treatment Group pooled analysis. Ann Oncol 23: 2919-2924, 2012.
8. Yokota M, Miyanaga K, Yonemura K, et al: Declining of memory functions of normal elderly persons. Psychiatry Clin Neurosci 54: 217-225, 2000.

9. Katoh S, Simogaki H, Onodera A, et al: Development of the revised version of Hasegawa's Dementia Scale (HDS-R). Jpn J Geriat Psychiatry 2: 1339-1347, 1991 (In Japanese).

10. Ball DL and Matthews JP: Prophylactic cranial irradiation: more questions than answers. Semin Radiat Oncol 5: 61-68, 1995.

11. Welzel T, Niethammer A, Mende U, et al: Diffusion tensor imaging screening of radiation-induced changes in the white matter after prophylactic cranial irradiation of patients with small cell lung cancer: first results of a prospective study. AJNR Am J Neuroradiol 29: 379-383, 2008.

12. Grosshans DR, Meyers CA, Allen PK, et al: Neurocognitive function in patients with small cell lung cancer: effect of prophylactic cranial irradiation. Cancer 112: 589-595, 2008.

13. Gondi V, Paulus R, Bruner DW, et al: Decline in tested and self-reported cognitive functioning after prophylactic cranial irradiation for lung cancer: pooled secondary analysis of Radiation Therapy Oncology Group randomized trials 0212 and 0214. Int J Radiat Oncol Biol Phys 86: 656-664, 2013.

14. Le Péchoux C, Dunant A, Senan S, et al; Prophylactic Cranial Irradiation (PCI) Collaborative Group: Standard-dose versus higher-dose prophylactic cranial irradiation (PCI) in patients with limited-stage small-cell lung cancer in complete remission after chemotherapy and thoracic radiotherapy (PCI 99-01, EORTC 22003-08004, RTOG 0212 and IFCT 99-01): a randomised clinical trial. Lancet Oncol 10: 467-474, 2009. 